\title{
The role of $\Delta 6$-desaturase acyl-carrier specificity in the efficient synthesis of long-chain polyunsaturated fatty acids in transgenic plants
}

\author{
Olga Sayanova, Noemi Ruiz-Lopez, Richard P. Haslam and Johnathan A. Napier* \\ Department of Biological Chemistry, Rothamsted Research, Harpenden, Herts, UK
}

Received 10 May 2011;

revised 19 July 2011;

accepted 21 July 2011.

*Correspondence (Tel +44 1582763133 ;

fax +44 1582 763010; email

johnathan.napier@rothamsted.ac.uk)
Keywords: desaturases, fatty acids, omega-3, polyunsaturated fatty acids, stearidonic acid, transgenic plants.

\begin{abstract}
Summary
The role of acyl-CoA-dependent $\Delta 6$-desaturation in the heterologous synthesis of omega-3 long-chain polyunsaturated fatty acids was systematically evaluated in transgenic yeast and Arabidopsis thaliana. The acyl-CoA $\Delta 6$-desaturase from the picoalga Ostreococcus tauri and orthologous activities from mouse (Mus musculus) and salmon (Salmo salar) were shown to generate substantial levels of $\Delta 6$-desaturated acyl-CoAs, in contrast to the phospholipid-dependent $\Delta 6$ desaturases from higher plants that failed to modify this metabolic pool. Transgenic plants expressing the acyl-CoA $\Delta 6$-desaturases from either 0 . tauri or salmon, in conjunction with the two additional activities required for the synthesis of $\mathrm{C} 20$ polyunsaturated fatty acids, contained higher levels of eicosapentaenoic acid compared with plants expressing the borage phospholipid-dependent $\Delta 6$-desaturase. The use of acyl-CoA-dependent $\Delta 6$-desaturases almost completely abolished the accumulation of unwanted biosynthetic intermediates such as $\gamma$-linolenic acid in total seed lipids. Expression of acyl-CoA $\Delta 6$-desaturases resulted in increased distribution of long-chain polyunsaturated fatty acids in the polar lipids of transgenic plants, reflecting the larger substrate pool available for acylation by enzymes of the Kennedy pathway. Expression of the 0 . tauri $\Delta 6$-desaturase in transgenic Camelina sativa plants also resulted in the accumulation of high levels of $\Delta 6$-desaturated fatty acids. This study provides evidence for the efficacy of using acyl-CoA-dependent $\Delta 6$-desaturases in the efficient metabolic engineering of transgenic plants with high value traits such as the synthesis of omega-3 LC-PUFAs.
\end{abstract}

\section{Introduction}

Fatty acid desaturases from vertebrates are generically classified as acyl-CoA desaturases (Okayasu et al., 1981; Sprecher and Chen, 1999), based on the assumption that these enzymes use CoA-linked fatty acids as their substrate. This substrate preference is assumed for both the stearate $\triangle 9$-desaturase SCD1 and also the 'front-end' cytochrome b5-fusion desaturases involved in the synthesis of long-chain polyunsaturates. However, the only example in the literature of the biochemical in vitro characterization of a front-end desaturase is for the rat 46 -desaturase (Okayasu et al., 1981). This enzyme was purified to homogeneity from rat liver microsomes and was used in detergent-containing assays in the presence of additional cytochrome $b 5$ and cytochrome $b 5$ reductase to show that linoleoyl-CoA was the reaction substrate. Surprisingly, the desaturase activity was absolutely dependent on the addition of exogenous cytochrome b5, although the subsequent cloning of the gene encoding the rat $\Delta 6$-desaturase (and other animal orthologs) revealed that the open reading frame contained an $\mathrm{N}$-terminalcytochrome $b 5$ domain expected to function as immediate electron donor (Cho et al., 1999; Marquardt et al., 2000). This sole study suggests that mammalian $\Delta 6$-desaturases use acyl-CoA substrates.

Biochemical studies with plants and fungi indicate a preference for lipid-linked substrates. Ground-breaking studies using alkenylether glycerolipids and tomato cell cultures demonstrated that purified plant microsomal $\Delta 12$ - and $\Delta 15$-desaturases act on (phospho)lipid-linked substrates (Sperling et al., 1993). In vitro enzyme assays with microsomes from the fungus Mucor circinelloides and from borage (Borago officinalis) indicated that $\Delta 6$ desaturases also act on the $s n-2$ position of phosphatidylcholine $(\mathrm{PC})$, converting linoleate at the $s n-2$ position of complex lipids to $\gamma$-linolenic acid (GLA, 18:3 $\Delta 6,9,15 ; \mathrm{n}-6)$ (Stymne and Stobart, 1986; Griffiths et al., 1988; Jackson et al., 1998). Because the purification of enzymatically active membrane-bound desaturases is notoriously difficult, the functional characterization of most front-end desaturases has been based on heterologous expression in various host organisms (usually S. cerevisiae) followed by appropriate fatty acid analysis. Using such approaches, it was shown (for example) that all $\Delta 6$-desaturases had similar specificity towards different fatty acid substrates, but that enzymes from different organisms differed with respect to the acyl-carrier (COA vs. phospholipids) they use as substrate (Domergue et al., 2003; Napier et al., 2003; Sato et al., 2004). These observations indicate approaches to engineer biosynthesis of omega-3 long-chain polyunsaturated fatty acids (LC-PUFAs) in plants (Drexler et al., 2003; Napier et al., 2004; Hoffmann et al., 2008; Petrie et al., 2010).

For example, detailed biochemical analyses of transgenic linseed expressing genes of the conventional $\Delta 6$-pathway ( $\Delta 6$ and $\Delta 5$-desaturases of the marine alga and $\Delta 6$-elongase from moss) indicated that successful production of non-native C20 LC-PUFAs in plants required efficient exchange of acyl groups 
between phospholipids and the acyl-CoA pool (Abbadi et al., 2004), due to the so-called substrate dichotomy arising from phospholipid-dependent desaturases and the acyl-CoA-dependent elongase that was predicted to generate a metabolic bottleneck (Domergue et al., 2005a; Napier, 2007; Robert, 2006). Approaches suggested to overcome this blockade (Damude and Kinney, 2008; Cheng et al., 2010; Petrie et al., 2010) include the use of acyl-CoA-dependent desaturases, thus allowing the synthesis of LC-PUFAs to proceed exclusively in the acyl-CoA pool. As noted earlier, mammalian desaturases have such substrate preference but the differences in codon-usage between plants and animals precluded the efficient expression of native animal sequences in transgenic plants. However, Domergue et al. (2005b) identified an acyl-CoA-dependent $\Delta 6$-desaturase from the picoalga Otreococcus tauri (OtD6). They noted the appearance of $\Delta 6$-desaturation products in the acyl-CoA pool prior to their presence in total fatty acids, consistent with acylCOA desaturation followed by incorporation of the COA-linked $\Delta 6$-desaturation product into phospholipids.

Here, we report on the efficacy of several different front-end $\Delta 6$-desaturases to produce LC-PUFAs in transgenic plants and yeast. Our data also indicate the unexpected consequences of changes to the acyl-CoA pool through the actions of acylCoA-dependent desaturases.

\section{Results and discussion}

\section{Systematic expression and analysis of $\Delta 6$-desaturases in} yeast

We set out to test whether the use of acyl-CoA-dependent desaturases in the biosynthesis of omega-3 LC-PUFAs can overcome the problem of substrate dichotomy. First, we examined the kinetics of in vivo desaturation for a number of different $\Delta 6$-desaturases previously shown to be active in yeast. The coding sequences for $\Delta 6$-desaturases from 0 . tauri OtD6 (Domergue et al., 2005b); mouse, MmD6 (Cho et al., 1999); salmon, SsD6 (Zheng et al., 2005); parasitic protozoa Trypanosoma brucei, TbD6 (Tripodi et al., 2006); borage, BoD6 (Sayanova et al., 1997) and Primula luteola, PID6 (Sayanova et al., 2006) were cloned into the yeast expression vector pYES2, and the accumulation of the $\Delta 6$-desaturation product stearidonic acid (SDA; 18:4 $46,9,12,15 ; n-3)$ was determined after a $24-h$ induction period (to allow the expression of the non-native desaturase) and the subsequent addition of the substrate fatty acid $\alpha$-linolenic acid (ALA; 18:3 $\Delta 9,12,15 \mathrm{n}$-3). Aliquots of the yeast cultures were taken at time points previously defined by Domergue et al. (2003), and trans-methylated and total fatty acid methyl esters (FAMes) were analyzed and quantitated by GC-FID. As can be seen in Figure S1, all six desaturases showed activity towards ALA, directing the synthesis of SDA in yeast. Of the six enzymes tested, the 0 . tauri $\Delta 6$-desaturase displayed the highest conversion (substrate to product) rate, followed by the mouse and salmon activities. Markedly lower conversation rates were observed for the trypanosome $\Delta 6$-desaturase and the two higher plant forms (Table 1). We detected SDA within 5 min for all of the non-higher plant enzymes (i.e. O. tauri, T. brucei, salmon and mouse) expressed in either our laboratory haploid yeast strain (W303) or the diploid INVSc1 (Invitrogen, Paisley, UK) strain used by Domergue et al. (2003, 2005b), who observed the accumulation of $\Delta 6$-desaturated fatty acids only after longer incubation.
Table 1 Desaturation efficiency of the $\Delta 6$-desaturases

\begin{tabular}{|c|c|c|c|}
\hline Construct & ALA & SDA & Conversion rate (\%) \\
\hline \multicolumn{4}{|l|}{ OtD6 } \\
\hline $5^{\prime}$ & $2.6 \pm 0.5$ & $2.6 \pm 0.1$ & 50.0 \\
\hline $1 \mathrm{~h}$ & $4.6 \pm 0.8$ & $7.7 \pm 1.0$ & 62.6 \\
\hline $24 \mathrm{~h}$ & $13.6 \pm 1.5$ & $36.5 \pm 2.1$ & 72.8 \\
\hline \multicolumn{4}{|l|}{ SsD6 } \\
\hline $5^{\prime}$ & $2.1 \pm 0.4$ & $0.6 \pm 0.1$ & 22.2 \\
\hline $1 \mathrm{~h}$ & $13.2 \pm 1.0$ & $4.3 \pm 0.8$ & 24.6 \\
\hline $24 \mathrm{~h}$ & $36.8 \pm 1.4$ & $22.9 \pm 2.9$ & 38.3 \\
\hline \multicolumn{4}{|l|}{ MmD6 } \\
\hline $5^{\prime}$ & $2.6 \pm 0.6$ & $0.3 \pm 0.1$ & 10.3 \\
\hline $1 \mathrm{~h}$ & $8.7 \pm 1.2$ & $2.1 \pm 0.7$ & 19.4 \\
\hline $24 \mathrm{~h}$ & $18.1 \pm 0.8$ & $12.1 \pm 3.2$ & 40.2 \\
\hline \multicolumn{4}{|l|}{ TbD6 } \\
\hline $5^{\prime}$ & $4.4 \pm 0.9$ & $0.3 \pm 0.1$ & 6.3 \\
\hline $1 \mathrm{~h}$ & $15.4 \pm 2.1$ & $1.2 \pm 0.2$ & 7.2 \\
\hline $24 \mathrm{~h}$ & $49.1 \pm 4.2$ & $7.2 \pm 1.1$ & 13.0 \\
\hline \multicolumn{4}{|l|}{ BoD6 } \\
\hline $5^{\prime}$ & $3.6 \pm 0.5$ & $0.2 \pm 0.1$ & 5.2 \\
\hline $1 \mathrm{~h}$ & $11.3 \pm 0.6$ & $0.5 \pm 0.2$ & 4.0 \\
\hline $24 \mathrm{~h}$ & $50.5 \pm 3.6$ & $3.6 \pm 0.5$ & 7.0 \\
\hline \multicolumn{4}{|l|}{ PID6 } \\
\hline $5^{\prime}$ & $3.2 \pm 0.5$ & 0.0 & - \\
\hline $1 \mathrm{~h}$ & $8.7 \pm 0.9$ & $0.2 \pm 01$ & 2.2 \\
\hline $24 \mathrm{~h}$ & $50.5 \pm 3.8$ & $3.6 \pm 0.3$ & 5.2 \\
\hline
\end{tabular}

Yeast transformed with respective constructs were grown for $24 \mathrm{~h}$ at $30^{\circ} \mathrm{C}$ in the presence of galactose before being supplemented with $250 \mu \mathrm{m} \alpha$ linolenic acid (ALA). Time points were taken $5 \mathrm{~min}, 1$ and $24 \mathrm{~h}$ after substrate was supplied to the medium. The ratios were calculated as (products/educt) using values corresponding to per cent of total fatty acids. Each value is the mean \pm SD from three independent experiments.

\section{Acyl-CoA profiling of $\Delta 6$-desaturase activities in yeast}

To better understand the dynamics of acyl desaturation in yeast, we also determined the acyl-CoA pool composition for the same time points. As can be seen in Figure 1, the $O$. tauri $\Delta 6$-desaturase generated substantial levels of SDA-CoA after just five minutes, although this was not directly proportional to the (higher) levels observed in total lipids at this time-point. Two other predicted acyl-CoA-dependent $\Delta 6$-desaturases, from mouse and salmon, also directed the conversion of ALA-COA to SDA-CoA within the first time-point $(5 \mathrm{~min})$, although again there was no obvious relationship between the levels of nonnative fatty acids in the acyl-COA pool vs. the total fatty acids. In similar analysis of the phospholipid-dependent borage $\Delta 6$-desaturase, only very minor levels of SDA were detected in either the acyl-COA pool or total fatty acids after $1 \mathrm{~h}$, despite the presence of high levels of substrate ALA. Thus, the early appearance of the $\Delta 6$-desaturation product SDA in the acylCOA pool is, in agreement with the studies of Domergue et al. $(2003,2005 b)$, indicative of an acyl-CoA-dependent desaturase. Conversely, the later detection of SDA-COA in yeast cells expressing BoD6 is commensurate with a lipid-dependent enzyme carrying out desaturation on fatty acids esterified to phospholipids, with these desaturation products then subsequently undergoing acyl-exchange and entering the COA pool. As noted earlier, several putative acyl-CoA-dependent 
OtD6

(a)

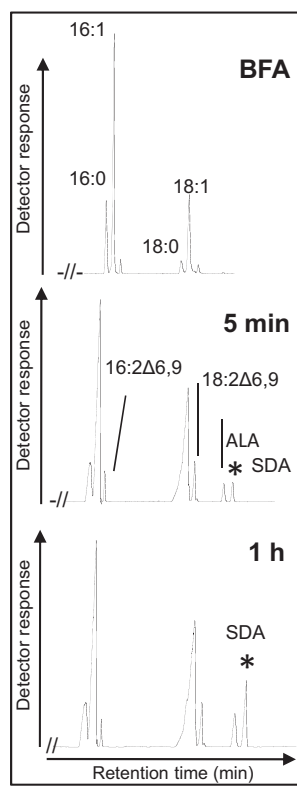

Total fatty acids

(b)

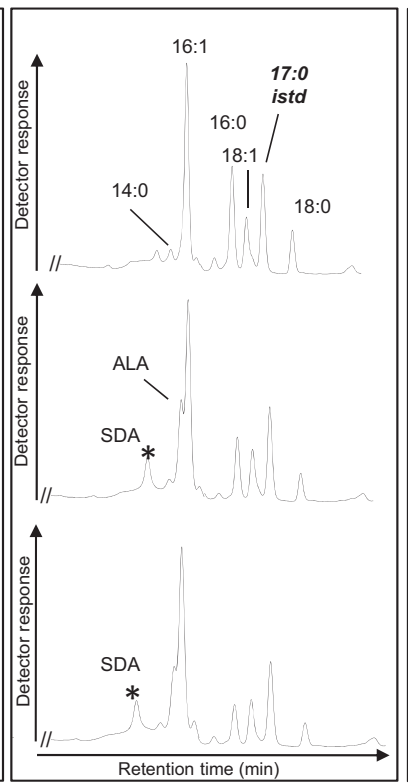

Acyl-CoA pool (c)

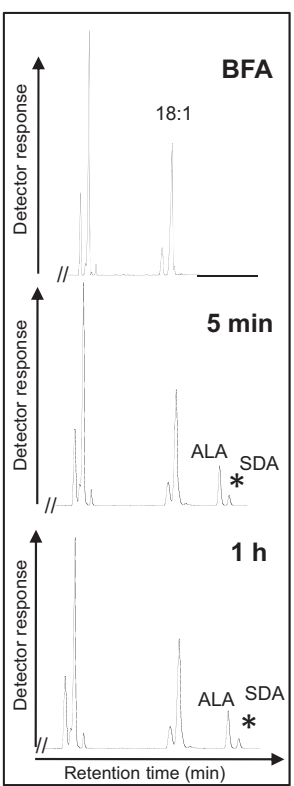

Total fatty acids
SsD6

(d)

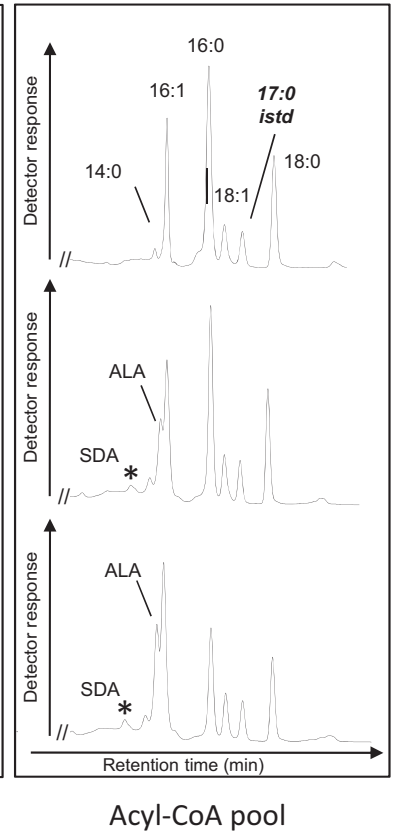

(e)

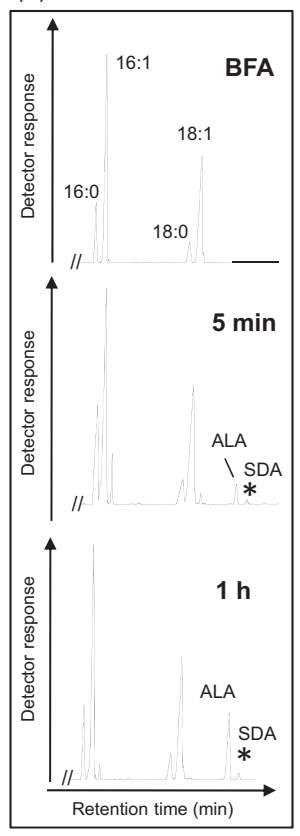

Total fatty acids

h
$\mathrm{MmD6}$

(f)
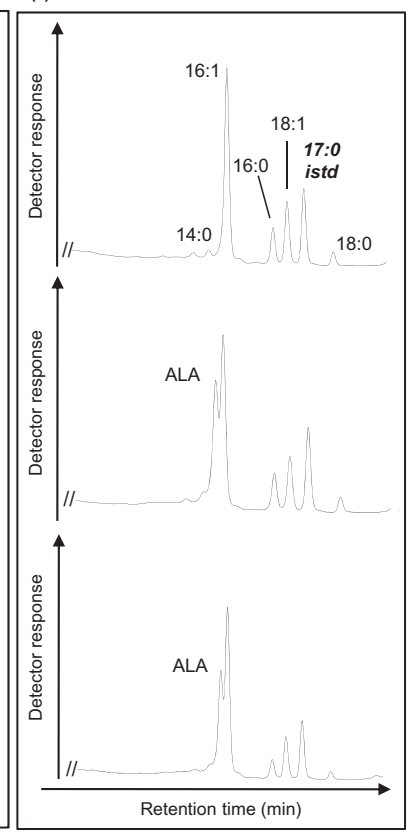

Acyl-CoA pool (g)

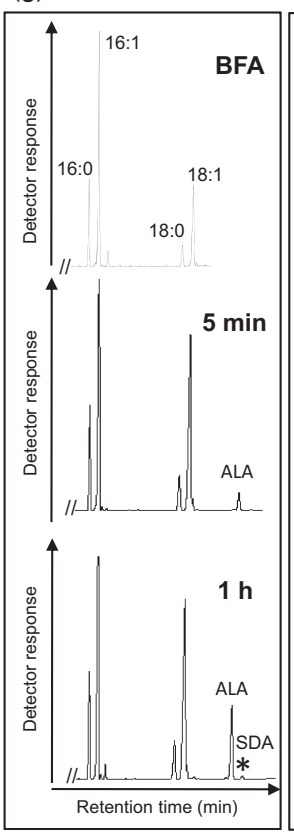

Total fatty acids
BoD6

(h)
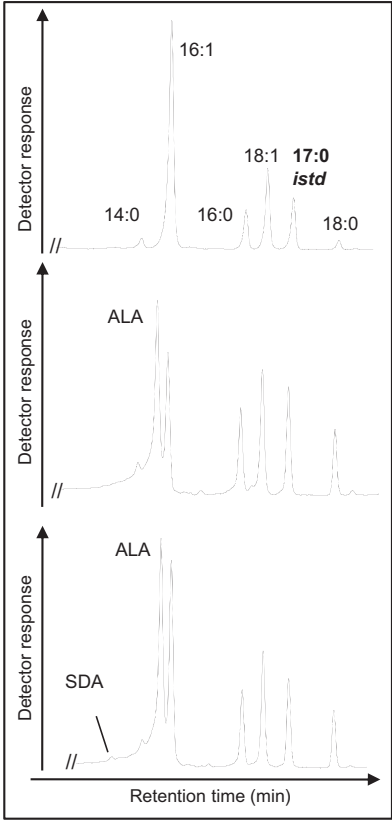

Acyl-CoA pool

Figure 1 Kinetic analysis of fatty acid profiles in lipids and acyl-CoAs of yeast expressing $\Delta 6$-desaturases in the presence of $\alpha$-linolenic acid (ALA). Cultures of yeast transformed with OtD6, SsD6, MmD6 and BoD6 were grown for $24 \mathrm{~h}$ at $30{ }^{\circ} \mathrm{C}$ in the presence of galactose before being supplemented with $250 \mu \mathrm{M}$ ALA. The fatty acid composition as well as the acyl-CoA pool composition of the transgenic yeast was analyzed before adding ALA (BFA = before addition) as well as $5 \mathrm{~min}$ and $1 \mathrm{~h}$ after substrate was supplied to the medium. The desaturated product stearidonic acid (SDA) is marked by a star. Data shown are representative for three independent experiments, with chromatographic resolution occurring against time ( $X$-axis). Detector response ( $Y$-axis) is derived from FID (a) or FLD with the excitation wavelength was set to $230 \mathrm{~nm}$ and the emission wavelength to $420 \mathrm{~nm}$.

(a) Fatty acid composition of yeast cells expressing OtD6 (a, b), SsD6 (c, d), MmD6 (e, f) and BoD6 (g, h) in the presence of ALA. (b) Acyl-CoA profiling of yeast cells expressing OtD6, MmD6, SsD6 and BoD6 in the presence of ALA. The internal standard $\left(I_{\text {std }}\right)$ is 17:0 acly-CoA. 
desaturases directed the accumulation of SDA in total fatty acids after only $5 \mathrm{~min}$. Given that the acyl-CoA pool is a very small metabolic pool (in the pmol range), we suggest that the observed accumulation represents the acyl-CoA-dependent incorporation of SDA into glycerolipids. This exchange between the acyl-CoA pool and phospholipids involves several enzymes, including the acyl-CoA-dependent enzymes of the Kennedy pathway, acyl-CoA generating activities such as phospholipases and also the reverse reaction of LPCAT (reviewed in Napier and Graham, 2010). The latter has been suggested as a mechanism by which the metabolic bottleneck of substrate dichotomy is overcome in vivo (Abbadi et al., 2004; Venegas-Calerón et al., 2010).

\section{Reconstitution of LC-PUFA synthesis in yeast}

To further explore the nature of these desaturases and better determine their acyl-substrate preferences, we co-expressed them with a microsomal acyl-CoA-dependent ketoacyl-CoA synthase (PSE1) from Physcomitrella patens, which has previously been shown to specifically elongate C18 $\Delta 6$-unsaturated acylCoAs to C20 forms (Zank et al., 2002). Here, we studied the desaturases from 0 . tauri (algal lower eukaryote acyl-CoAdependent enzyme) salmon (vertebrate acyl-CoA enzyme) and borage (higher plant phospholipid-dependent enzyme). As the product of an acyl-CoA-dependent desaturase will remain esterified to COA, the in vivo coupling of desaturation and elongation has previously been used as an (indirect) method by which to differentiate desaturase substrate preference (COA vs. phospholipids) (Domergue et al., 2003). Co-expression in yeast, induction and kinetic analysis of total fatty acids and acyl-CoAs was carried out as described earlier. As can be seen in Figure 2, when OtD6 was co-expressed with PSE1, substantial levels of both the $\Delta 6$-desaturation product SDA and the subsequent C20 elongation product eicosatetraenoic acid (ETA; 20:4n-3) were detected in the acyl-CoA pool after only 5 min, whereas they comprised $<2 \%$ of the yeast total fatty acids at this time (Table 2). The absolute levels of SDA-COA and ETA-CoA did not substantially increase over $24 \mathrm{~h}$ for OtD6 + PSE1, whereas their percentage of total fatty acids (tfa) increased markedly (Figure 2, Table 2). Co-expression of SsD6 with PSE1 generated modest but detectable levels of SDA-COA and ETA-COA (Figure 2), and the tfa profile showed slower accumulation of SDA with lower levels of ETA (Table 2). As predicted for a phospholipid-dependent desaturase, BoD6 + PSE1 did not generate any appreciable SDA-COA or ETA-COA, although both these non-native fatty acids were present in tfas.
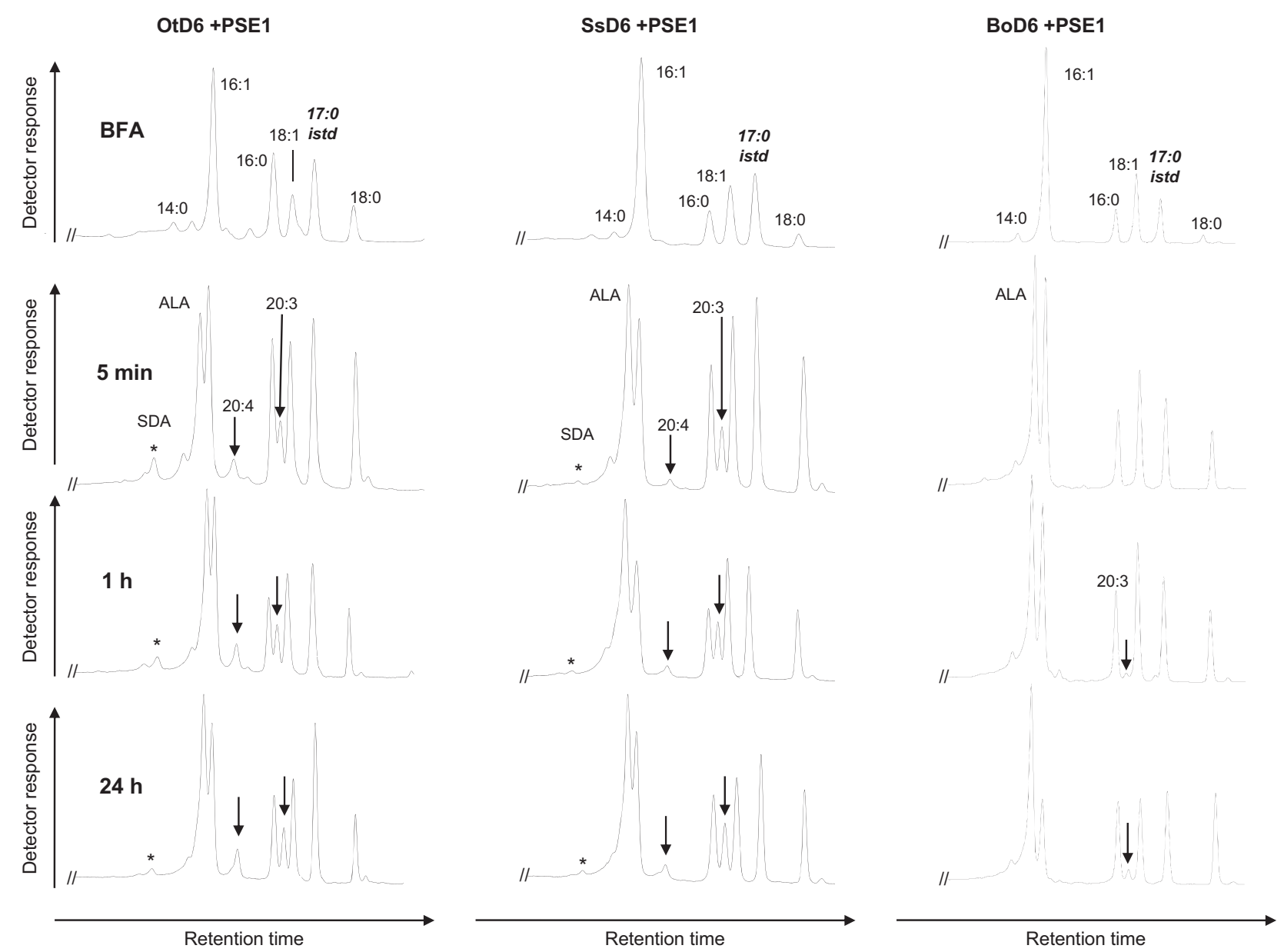

Figure 2 Kinetic analysis of fatty acid changes in acyl-CoAs of yeast co-expressing $\Delta 6$-desaturases and PSE1. Cultures of yeast co-transformed with one of the $\Delta 6$-desaturases (OtD6, SsD6 or BoD6) and PSE1 were grown for $24 \mathrm{~h}$ at $30{ }^{\circ} \mathrm{C}$ in the presence of galactose before being supplemented with $250 \mu \mathrm{M} \alpha$-linolenic acid (ALA). The acyl-COA pool composition of the transgenic yeast was analyzed before adding ALA (BFA) and as well as $5 \mathrm{~min}, 1$ and $24 \mathrm{~h}$ after substrate was supplied to the medium. The desaturated product stearidonic acid (SDA) is marked by a star, and elongated products are indicated with arrows. Data shown are representative for three independent experiments. The internal standard (Istd) is 17:0 acly-CoA. 
Table 2 Kinetic analysis of fatty acid changes of yeast co-expressing $\Delta 6$-desaturases and PSE1

\begin{tabular}{lccc}
\hline Construct & $18: 3 \mathrm{n}-3(\mathrm{ALA})$ & $18: 4 \mathrm{n}-3(\mathrm{SDA})$ & $20: 4 \mathrm{n}-3$ (ETA) \\
\hline OtD6 + PSE1 & & & \\
$5^{\prime}$ & $1.4 \pm 0.4$ & $1.2 \pm 0.3$ & $<0.5$ \\
$1 \mathrm{~h}$ & $4.2 \pm 1.0$ & $1.9 \pm 0.2$ & $1.0 \pm 0.3$ \\
$24 \mathrm{~h}$ & $17.7 \pm 2.5$ & $8.2 \pm 0.9$ & $6.2 \pm 0.7$ \\
SsD6 + PSE1 & & & \\
$5^{\prime}$ & $1.0 \pm 0.2$ & - & - \\
$1 \mathrm{~h}$ & $6.5 \pm 1.1$ & $0.4 \pm 0.1$ & $0.7 \pm 0.2$ \\
$24 \mathrm{~h}$ & $32.0 \pm 4.6$ & $6.1 \pm 1.0$ & $1.3 \pm 0.3$ \\
BoD6 + PSE1 & & & - \\
$5^{\prime}$ & $2.1 \pm 0.5$ & - & $0.3 \pm 0.1$ \\
$1 \mathrm{~h}$ & $10.2 \pm 2.2$ & $0.2 \pm 0.1$ & $1.5 \pm 0.6$ \\
$24 \mathrm{~h}$ & $40.6 \pm 4.4$ & $0.5 \pm 0.2$ & \\
\hline
\end{tabular}

Cultures of yeast co-transformed with one of the $\Delta 6$-desaturases (OtD6, SsD6 or BoD6) and PSE1 were grown for $24 \mathrm{~h}$ at $30{ }^{\circ} \mathrm{C}$ in the presence of galactose before being supplemented with $250 \mu \mathrm{m}$ ALA. The fatty acid composition of the transgenic yeast was analyzed $5 \mathrm{~min}, 1$ and $24 \mathrm{~h}$ after substrate was supplied to the medium. Each value is the mean \pm SD from three independent experiments.

ALA, $\boldsymbol{\alpha}$-linolenic acid; SDA, stearidonic acid; ETA, eicosatetraenoic acid.

Interestingly, an abundant acyl-CoA identified as 20:3n-3COA was present in all samples in which ALA had been exogenously supplied (Figure 2), indicating that PSE1 can utilize additional C18 substrates beyond just SDA and GLA. In view of the promiscuous activity of the PSE1 $\Delta 6$-elongase, which potentially could deplete the substrate pool of ALA-CoA, we repeated the co-expression experiments with a second, different 46-elongase, CeElo6 from Caenorhabditis elegans (Beaudoin et al., 2000; Napier and Michaelson, 2001; Robert et al., 2005), which is specific for C18 $\Delta 6$-unsaturated substrates. Desaturation and elongation products were almost undetectable in the acyl-CoA pool of yeast cells expressing the borage desaturase and CeElo6 (Table S1).

Domergue et al. (2005b) suggested the asynchronous appearance of desaturation products in the acyl-CoA pool prior to detection in tfa extracts (as they reported for the 0 . tauri $\Delta 6$-desaturase) as a diagnostic hallmark of a bona fide acylCoA-dependent desaturase. Our results indicate that this is not a reliable indicator. Also, the presence or absence of desaturation products in total lipid extracts is not in itself likely to prove a reliable marker for enzyme substrate preference. Some small differences between our present study and that of Domergue et al. (2003, 2005b), including the detection of acyl-CoA intermediates for OtD6 + PSE1 and SSD6 + PSE1 reactions, likely reflect improvements in chromatographic resolution of acylCoAs. Collectively our data support the classification of OtD6 and SsD6 as acyl-CoA-dependent activities.

\section{$\Delta 6$-desaturase activities in transgenic plants}

Previously, no systematic comparative analysis of the performance of different $\Delta 6$-desaturases in transgenic plants has been carried out, even though the use of acyl-CoA-dependent $\Delta 6$-desaturases has been widely suggested as a means to overcome the metabolic bottleneck of substrate dichotomy, and hence, enhance the accumulation of valuable C20+ long-chain
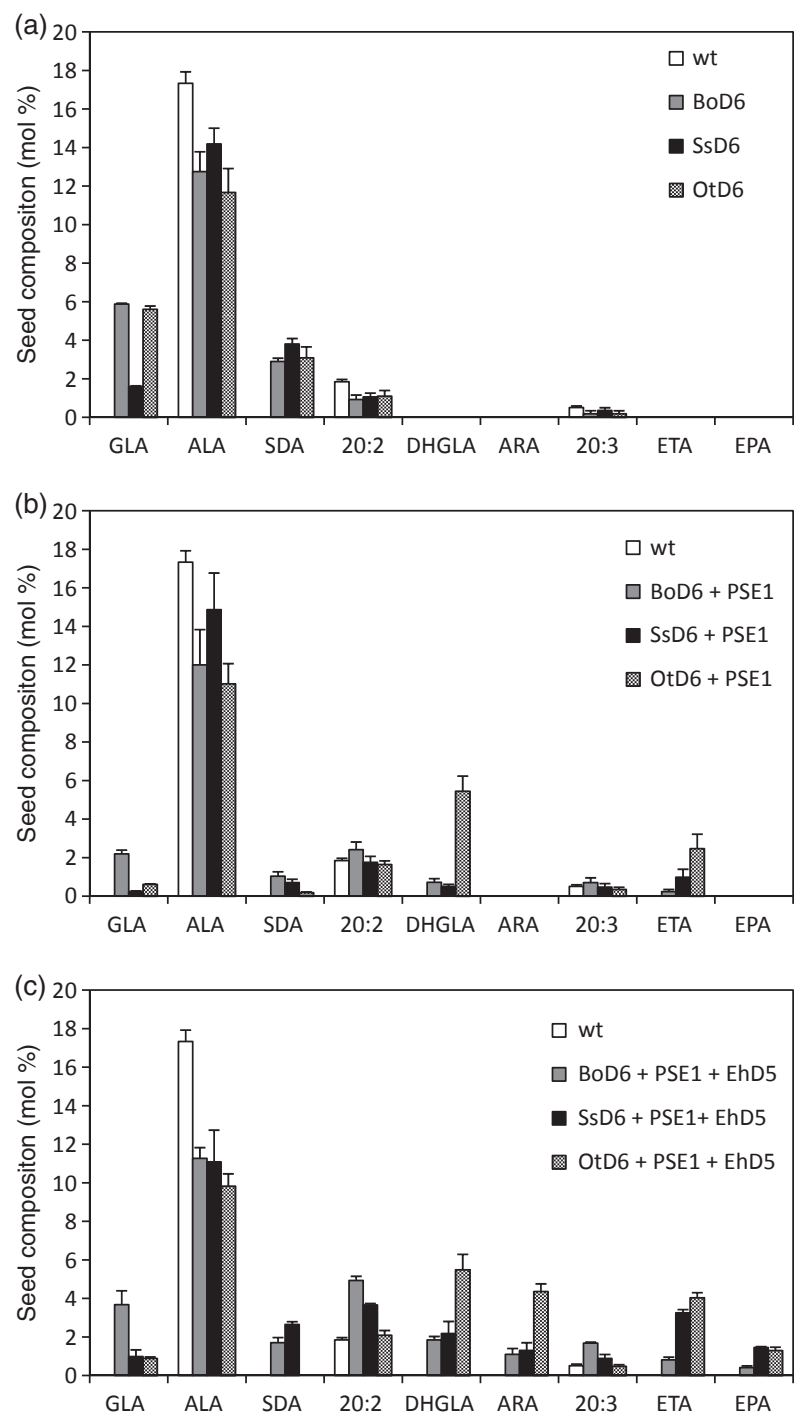

Figure 3 Total fatty acid composition of seeds from transgenic Arabidopsis lines. FAMEs were analyzed from wild type (wt), single-transgenic lines expressing $\Delta 6$-desaturases (a), double-transgenic lines co-expressing $\Delta 6$-desaturases and PSE1 (b) and triple-transgenic lines of Arabidopsis, co-expressing $\Delta 6$-desaturases, PSE1 and EhD5 (c). Each value represents the mean \pm SD of 10 independent lines. In all cases, the SD was $<1$.

polyunsaturated fatty acids. We therefore generated transgenic Arabidopsis plants that expressed OtD6, SsD6 or BoD6 desaturases under the control of the conglycinin promoter. As shown in Figure 3, all three desaturases independently directed the synthesis and accumulation of $\Delta 6$-unsaturated fatty acids in mature seeds from homozygous T2 progeny. SDA accumulated to broadly similar levels for all three desaturases (3.5\% tfa, BoD6, 4.7\% tfa, SsD6; 3.8\% tfa, OtD6), whereas plants expressing SsD6 accumulated less GLA (1.8\% tfa) compared with those expressing BoD6 or OtD6 (6.0 and 5.8\% tfa). These data indicate that the salmon desaturase has a strong substrate preference for omega-3 fatty acids. Petrie et al. (2010) recently reported that expression of an acyl-CoA-dependent $\Delta 6$-desaturase from Micromonas pusilla yielded GLA + SDA levels ranging from $8 \%$ to $18 \%$ tfa in the seeds of transgenic Arabidopsis. 
The $M$. pusilla $\Delta 6$-desaturase appears to have a preference for omega-3 substrate, unlike the OtD6 enzyme that has a clear omega-6 bias.

In a second iteration, the individual desaturases were co-expressed with the PSE1 $\Delta 6$-elongase, also under the control of the seed-specific conglycinin promoter. Similar analysis fatty acid composition in mature T2 seeds confirmed our observations in yeast that the phospholipid-dependent BoD6 desaturase did not facilitate the subsequent elongation of $\Delta 6$-unsaturated fatty acids, with only low levels of C20 products being detected $(1.0 \%$ 20:3n-6, $0.4 \% 20: 4 n-3)$. Seed-specific expression of SsD6 + PSE1 yielded $0.8 \% \quad 20: 3 n-6$ and $1.5 \% \quad 20: 4 n-3$, and OtD6 + PSE1 generated 5.8\% 20:3n-6 and 3.0\% 20:4n-3, indicative (most clearly in the latter case) of a more efficient flux of substrate between the desaturation and elongation reactions.

In a third iteration, we aimed to reconstruct the biosynthetic pathway for C20 LC-PUFAs by co-expressing the $\Delta 6$-desaturases and the PSE1 $\Delta 6$-elongase with a downstream C20 $\Delta 5$-desaturase, thus reconstructing the biosynthetic pathway for C20 LC-PUFAs. We placed the recently characterized Emiliania huxleyi $\Delta 5$-desaturase (EhD5) (Sayanova et al., 2011). Low levels of EPA were obtained in mature T2 seeds $0.5 \%$ tfa for BoD 6 + PSE1 + EhD5; 1.4\% tfa for OtD6 + PSE1 + EhD5; $1.5 \%$ tfa for SsD6 + PSE1 + EhD5). Interestingly, the omega-6 LC-PUFA arachidonic acid (ARA, 20:4n-6) accumulated to $4.7 \%$ tfa in the OtD6-containing construct, compared with $1.2 \% \mathrm{tfa}$ in the
BoD6-containing construct and $1.2 \%$ in the SsD6-containing version, perhaps indicative of an omega- 6 substrate preference with this enzyme.

\section{Targeted lipidomic analysis of transgenic plants expressing $\Delta 6$-desaturases}

To better define the biochemical basis of these results, detailed analysis was carried out on the acyl-CoA pool and glycerolipids of these Arabidopsis seeds. As can be seen in Figure 4 and confirming previous observations, the acyl-CoA pool is altered by the expression of heterologous desaturases and elongase. In the case of the transgenic lines solely expressing a $\Delta 6$-desaturase, acyl-CoA profiles for OtD6 and SsD6 contained an additional peak identified as SDA-COA, whereas this peak was absent in profiles for BoD6, which was essentially the same as WT (not shown). Co-expression of the PSE1 elongase with the $\Delta 6$-desaturase confirmed the promiscuous nature of the PSE1 activity, as a peak identified as 20:2n-6 was present in all three double constructs. In the case of BoD6 + PSE1, a small peak identified as 20:3 (n-3 or n-6; it is not possible resolve regioisomers using chromatography) was detected. This peak was not seen in the OtD6 + PSE1 or SSD6 + PSE1 double constructs (Figure 4). In the case of the OtD6 and SsD6 double constructs, an additional acyl-CoA identified as 20:4-CoA was interpreted as the elongation product of SDA-COA. In support of this, the levels of SDA-CoA were seen to decrease in these samples. For seeds derived from the triple construct, in which a $\Delta 6$-desaturase and (a)
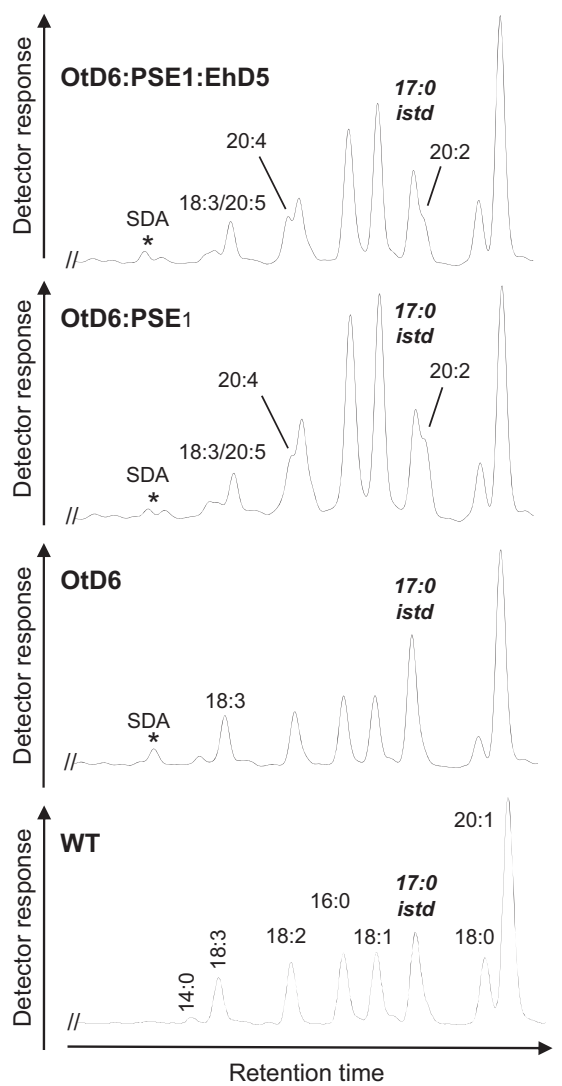

(b)
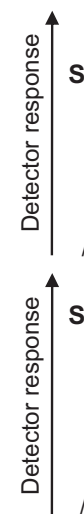

0
0
0
0
0
0
0
0
$\overline{0}$
0
0
0
0

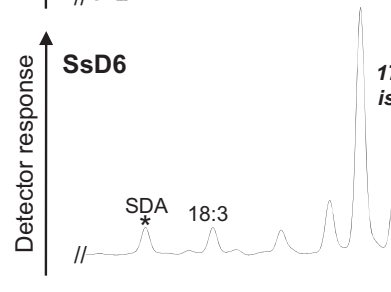

(c)
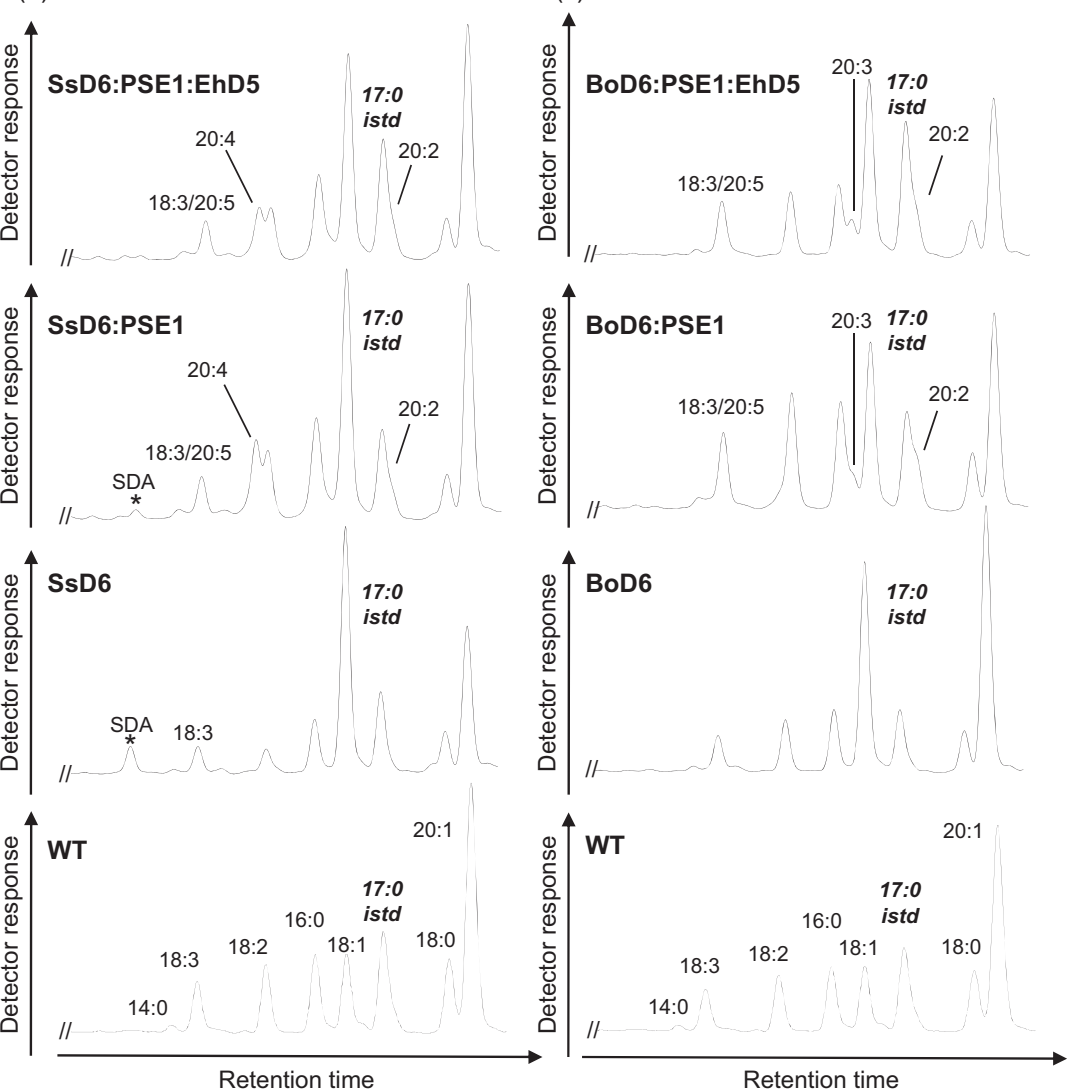

Figure 4 Acyl-CoA profiles of transgenic Arabidopsis. Acyl-CoA profiles of developing seeds of wild type (wt) and single-, double- and triple-transgenic Arabidopsis lines, expressing Otd6 (a), SsD6 (b) and BoD6 (c), The internal standard ( $\left.I_{\mathrm{std}}\right)$ is 17:0 acyl-CoA. The positions of transgene-derived nonnative fatty acids are indicated. 
PSE1 were combined with EhD5, the acyl-CoA profiles were essentially the same as those observed with the double construct (Figure 4), with an apparent conversion of heterologous SDA-CoA to 20:4-CoA. Again, due to the limitations of the chromatography used to resolve acyl-CoAs, it was not possible to determine whether this 20:4-CoA was ARA (20:4n-6, from the elongation and $\triangle 5$-desaturation of GLA) or $20: 4 n-3$ (the elongation product of SDA).

The same seed samples for the single and double constructs were also subject to detailed analysis of glycerolipids (GL) and glycerophospholipids (Tables 3 and 4). In the cases of the individual desaturase constructs, this revealed the widespread distribution of $\Delta 6$-desaturation products amongst polar and neutral lipids, with GLA and SDA being present, at varying levels, in triacylglycerol (TAG), diacylglycerol (DAG) and phospholipids (PL). Interestingly, the ratio of GLA in neutral lipids (NL) vs. phospholipids (PL) was higher from a lipid-dependent desaturase BoD6 (5.6\% in NL and $4.0 \%$ in $\mathrm{PL})$, than from SsD6 (1.6\% in NL and $3.7 \%$ in $\mathrm{PL}$ ) or OtD6 (5.4\% in NL and $10.9 \%$ in $\mathrm{PL}$ ) (Figure 5). Unexpectedly for acyl-CoA-dependent desaturases OtD6 and SsD6, GLA was accumulated mainly in polar lipids [phosphoethanolamine $(\mathrm{PE})$; phosphatidycholine $(\mathrm{PC})$ ]. SDA was present in
NL (2.6\%) and PL (1.25\%) for BoD6-expressing seeds, at 3.2\% in both $\mathrm{NL}$ and PL for OtD6 and higher in NL (3.7\%) than PL (2.5\%) for SsD6, thus demonstrating that salmon $\Delta 6$-desaturase has preference for $n-3$ substrates. Similar analysis was also carried out for the double constructs described earlier. Here, the combination of the OtD6 desaturase and PSE1 elongase resulted in the specific enrichment $(9.8 \% \mathrm{tfa})$ of DHGLA in PC, compared with $4.8 \%$ tfa in DAG and $3.4 \%$ for PI (Table 4 ). A similar distribution (albeit at a lower baseline) was also observed for SSD6 + PSE1, with notable accumulation of DHGLA in PC and to a lesser extent in PE. In contrast, DHGLA generated by BoD6 + PSE1 accumulated more in all phospholipids compared with neutral lipids, although to an overall lower level.

\section{Expression of the acyl-CoA-dependent $\Delta 6$-desaturase OtD6 in transgenic Camelina sativa}

To compare with our observations in Arabidopsis, we also engineered Camelina sativa to express the OtD6 acyl-CoA desaturase (using a similar construct to that used in Arabidopsis). C. sativa is a (re-)emerging oilseed crop, grown extensively in North America as a biofuel crop due to its modest requirements for nitrogen

Table 3 Fatty acid composition (mol\%) of neutral lipids isolated from seeds of wild type (wt), single- and double-transgenic Arabidopsis lines

\begin{tabular}{|c|c|c|c|c|c|c|c|c|c|c|c|}
\hline Mol\% & $16: 0$ & $18: 0$ & $18: 1$ & LA & GLA & ALA & SDA & $20: 1$ & DHGLA & ETA & Others \\
\hline \multicolumn{12}{|c|}{ Total neutral lipids } \\
\hline$w t$ & 7.1 & 3.5 & 16.3 & 27.4 & 0.0 & 17.2 & 0.0 & 21.2 & 0.0 & 0.0 & 7.2 \\
\hline BoD6 & 8.4 & 3.9 & 23.0 & 21.5 & 5.6 & 12.2 & 2.6 & 18.3 & 0.0 & 0.0 & 4.4 \\
\hline SsD6 & 8.6 & 3.9 & 20.2 & 27.3 & 1.6 & 14.3 & 3.7 & 15.2 & 0.0 & 0.0 & 5.2 \\
\hline OtD6 & 8.5 & 3.4 & 17.1 & 26.3 & 5.4 & 12.1 & 3.2 & 18.8 & 0.0 & 0.0 & 5.1 \\
\hline SsD6 + PSE1 & 8.5 & 3.8 & 17.8 & 29.6 & $<0.5$ & 15.6 & 0.8 & 15.9 & 0.5 & 1.1 & 6.3 \\
\hline OtD6 + PSE1 & 9.2 & 3.8 & 15.5 & 28.8 & 0.6 & 11.4 & $<0.5$ & 16.6 & 5.8 & 2.8 & 5.5 \\
\hline \multicolumn{12}{|l|}{ TAG } \\
\hline wt & 8.2 & 3.5 & 16.4 & 27.3 & 0.0 & 17.4 & 0.0 & 20.3 & 0.0 & 0.0 & 6.9 \\
\hline BoD6 & 8.2 & 3.8 & 22.3 & 21.6 & 5.8 & 13.0 & 3.0 & 17.8 & 0.0 & 0.0 & 4.6 \\
\hline SsD6 & 8.6 & 3.8 & 20.3 & 27.2 & 1.6 & 14.3 & 3.7 & 15.3 & 0.0 & 0.0 & 5.2 \\
\hline BoD6 + PSE1 & 9.0 & 3.6 & 18.0 & 28.7 & 2.3 & 12.7 & 1.1 & 17.1 & 0.7 & $<0.5$ & 6.9 \\
\hline SSD6 + PSE1 & 8.7 & 3.8 & 18.4 & 29.6 & $<0.5$ & 14.9 & 0.7 & 16.3 & 0.5 & 1.0 & 6.1 \\
\hline OtD6 + PSE1 & 9.2 & 3.8 & 15.8 & 28.9 & 0.6 & 11.3 & $<0.5$ & 16.8 & 5.6 & 2.6 & 5.5 \\
\hline \multicolumn{12}{|l|}{ DAG } \\
\hline wt & 7.8 & 3.6 & 17.1 & 36.1 & 0.0 & 14.4 & 0.0 & 14.7 & 0.0 & 0.0 & 6.2 \\
\hline BoD6 & 8.7 & 3.8 & 24.8 & 24.5 & 5.1 & 11.1 & 2.4 & 15.2 & 0.0 & 0.0 & 4.3 \\
\hline SsD6 & 8.6 & 3.7 & 19.3 & 32.2 & 2.2 & 12.4 & 4.4 & 12.1 & 0.0 & 0.0 & 5.0 \\
\hline OtD6 & 8.7 & 3.3 & 19.2 & 28.8 & 6.6 & 10.3 & 3.4 & 14.6 & 0.0 & 0.0 & 5.1 \\
\hline BoD6 + PSE1 & 10.3 & 3.8 & 18.5 & 30.9 & 1.8 & 11.1 & 0.9 & 13.9 & 1.2 & 0.5 & 7.1 \\
\hline SsD6 + PSE1 & 8.7 & 3.5 & 17.8 & 33.5 & $<0.5$ & 14.6 & 1.3 & 11.8 & 0.7 & 1.4 & 6.8 \\
\hline OtD6 + PSE1 & 9.9 & 3.9 & 18.5 & 31.2 & 0.7 & 9.7 & $<0.5$ & 12.8 & 4.8 & 2.2 & 6.4 \\
\hline \multicolumn{12}{|l|}{ Free fatty acids } \\
\hline wt & 9.5 & 8.5 & 15.5 & 22.1 & 0.0 & 12.7 & 0.0 & 21.7 & 0.0 & 0.0 & 9.9 \\
\hline BoD6 & 9.9 & 7.2 & 18.4 & 17.1 & 5.4 & 12.3 & 3.2 & 20.6 & 0.0 & 0.0 & 6.0 \\
\hline BoD6 + PSE1 & 13.1 & 6.9 & 15.2 & 21.7 & 2.1 & 9.5 & 0.9 & 20.6 & 0.7 & $<0.5$ & 9.3 \\
\hline SsD6 + PSE1 & 11.8 & 6.8 & 14.5 & 22.2 & 0.6 & 14.8 & 1.0 & 17.9 & 0.7 & 1.4 & 8.4 \\
\hline OtD6 + PSE1 & 11.2 & 6.3 & 11.9 & 23.5 & 0.9 & 11.6 & $<0.5$ & 18.5 & 5.6 & 2.8 & 7.6 \\
\hline
\end{tabular}

Values shown are means derived from independent lines $(n=5)$. In all cases, SD was $<1$.

GLA, $\gamma$-linolenic acid; ALA, $\alpha$-linolenic acid; SDA, stearidonic acid; ETA, eicosatetraenoic acid. 
Table 4 Fatty acid composition (mol\%) of polar lipids isolated from seeds of wild type (wt), single- and double-transgenic Arabidopsis lines

\begin{tabular}{|c|c|c|c|c|c|c|c|c|c|c|c|}
\hline & $16: 0$ & $18: 0$ & 18:1 & LA & GLA & ALA & SDA & $20: 1$ & DHGLAA & ETA & Others \\
\hline \multicolumn{12}{|l|}{ Total polar lipids } \\
\hline wt & 11.0 & 2.7 & 14.5 & 49.2 & 0.0 & 12.3 & 0.0 & 5.0 & 0.0 & 0.0 & 5.3 \\
\hline BoD6 & 14.5 & 2.6 & 24.0 & 39.3 & 4.0 & 8.3 & 1.2 & 4.2 & 0.0 & 0.0 & 1.8 \\
\hline SsD6 & 15.2 & 2.9 & 17.3 & 43.7 & 3.7 & 7.5 & 2.5 & 3.9 & 0.0 & 0.0 & 3.3 \\
\hline OtD6 & 14.2 & 2.4 & 17.9 & 36.7 & 10.9 & 7.3 & 3.2 & 4.9 & 0.0 & 0.0 & 2.4 \\
\hline BoD6 + PSE1 & 14.1 & 2.4 & 19.1 & 47.1 & 0.8 & 8.1 & $<0.5$ & 3.3 & 2.0 & $<0.5$ & 3.0 \\
\hline SsD6 + PSE1 & 12.6 & 3.2 & 16.0 & 41.0 & 1.9 & 8.2 & 2.5 & 6.8 & 1.9 & 1.4 & 4.3 \\
\hline OtD6 + PSE1 & 13.7 & 2.4 & 19.2 & 40.4 & $<0.5$ & 6.3 & $<0.5$ & 4.1 & 8.5 & 1.8 & 3.6 \\
\hline \multicolumn{12}{|l|}{ PC } \\
\hline wt & 8.8 & 7.7 & 15.6 & 41.1 & 0.0 & 11.3 & 0.0 & 6.7 & 0.0 & 0.0 & 8.7 \\
\hline BoD6 & 9.8 & 2.2 & 27.5 & 39.6 & 3.9 & 8.8 & 1.2 & 4.6 & 0.0 & 0.0 & 2.3 \\
\hline SsD6 & 10.2 & 2.3 & 20.4 & 45.7 & 3.7 & 7.8 & 2.7 & 4.2 & 0.0 & 0.0 & 2.9 \\
\hline OtD6 & 10.0 & 2.1 & 20.9 & 37.4 & 11.4 & 7.3 & 3.3 & 4.9 & 0.0 & 0.0 & 2.7 \\
\hline BoD6 + PSE1 & 11.1 & 2.5 & 20.2 & 44.6 & 0.7 & 9.0 & $<0.5$ & 5.1 & 2.0 & 0.5 & 4.3 \\
\hline SSD6 + PSE1 & 10.5 & 2.4 & 19.6 & 45.9 & $<0.5$ & 9.2 & $<0.5$ & 4.4 & 2.1 & 1.3 & 4.8 \\
\hline OtD6 + PSE1 & 10.5 & 2.1 & 20.7 & 38.6 & $<0.5$ & 6.7 & $<0.5$ & 5.0 & 9.8 & 2.2 & 4.3 \\
\hline \multicolumn{12}{|l|}{$\mathrm{PE}$} \\
\hline wt & 13.3 & 2.5 & 12.0 & 53.4 & 0.0 & 10.5 & 0.0 & 2.9 & 0.0 & 0.0 & 5.3 \\
\hline BoD6 & 16.9 & 2.1 & 19.0 & 44.2 & 5.0 & 7.6 & 1.2 & 2.6 & 0.0 & 0.0 & 1.5 \\
\hline SsD6 & 16.2 & 2.7 & 15.4 & 46.6 & 4.2 & 7.0 & 2.1 & 3.4 & 0.0 & 0.0 & 2.5 \\
\hline OtD6 & 17.5 & 2.2 & 14.9 & 40.8 & 11.5 & 6.2 & 2.3 & 2.6 & 0.0 & 0.0 & 2.0 \\
\hline BoD6 + PSE1 & 18.6 & 2.0 & 14.7 & 48.9 & 1.2 & 7.4 & $<0.5$ & 2.2 & 2.1 & 0.5 & 2.4 \\
\hline SSD6 + PSE1 & 17.8 & 2.2 & 13.5 & 50.3 & $<0.5$ & 8.3 & $<0.5$ & 2.4 & 1.6 & 1.1 & 2.8 \\
\hline OtD6 + PSE1 & 19.1 & 2.2 & 14.2 & 44.6 & $<0.5$ & 5.9 & 0.0 & 2.5 & 7.3 & 1.8 & 2.4 \\
\hline \multicolumn{12}{|l|}{$\mathrm{Pl}$} \\
\hline wt & 24.9 & 5.2 & 8.7 & 38.5 & 0.0 & 16.1 & 0.0 & 1.9 & 0.0 & 0.0 & 4.8 \\
\hline BoD6 & 28.9 & 4.5 & 12.3 & 32.3 & 3.2 & 8.8 & 1.3 & 5.5 & 0.0 & 0.0 & 3.1 \\
\hline SsD6 & 29.8 & 4.5 & 9.6 & 34.5 & 2.5 & 8.7 & 2.4 & 4.9 & 0.0 & 0.0 & 3.1 \\
\hline OtD6 & 30.5 & 4.0 & 9.6 & 30.7 & 7.6 & 7.4 & 2.2 & 4.8 & 0.0 & 0.0 & 3.2 \\
\hline BoD6 + PSE1 & 29.0 & 5.1 & 10.9 & 36.6 & 0.9 & 8.0 & $<0.5$ & 3.1 & 2.3 & 0.9 & 3.3 \\
\hline SSD6 + PSE1 & 29.3 & 4.6 & 9.9 & 35.4 & $<0.5$ & 10.0 & $<0.5$ & 3.8 & 0.9 & 1.0 & 5.1 \\
\hline OtD6 + PSE1 & 30.8 & 4.8 & 10.7 & 34.9 & 0.8 & 7.2 & $<0.5$ & 3.9 & 2.5 & 1.0 & 3.4 \\
\hline
\end{tabular}

Values shown are means derived from independent lines $(n=5)$. In all cases, SD was $<1$.

GLA, $\gamma$-linolenic acid; ALA, $\alpha$-linolenic acid; SDA, stearidonic acid; ETA, eicosatetraenoic acid; DHGLA = di-homo- $\gamma$-linolenic acid.

(Gehringer et al., 2006) but also used in Europe as a high omega-3 vegetable oil for human nutrition. As shown in Table 5, we recovered 12 transgenic $C$. sativa lines, the seeds of which were shown to contain substantial levels of GLA ( 18\% tfa) and SDA ( $\sim 12 \% \mathrm{tfa})$. The level of non-native $\Delta 6$-desaturated fatty acids (30\% of seed oil) was about twice that achieved in Arabidopsis using the same construct (Figure 3a) and did not compromise $C$. sativa seed development or germination.

The results obtained with $C$. sativa for the acyl-CoA-dependent OtD6 desaturase were compared well to previous attempts to synthesize these fatty acids in transgenic soybean (Sato et al., 2004; Eckert et al., 2006) and Brassica juncea (Hong et al., 2002) using phospholipid-dependent $\Delta 6$-desaturase activities. Compared with the accumulation of GLA and SDA in transgenic Arabidopsis seeds, the levels achieved in $C$. sativa with the same acyl-CoA-dependent OtD6 are notably higher (cf. Figure 3a maximal total levels of $\Delta 6$-desaturated fatty acids $=\sim 15 \%$ ). In this current study, the levels of ALA are markedly reduced in C. sativa lines accumulating GLA and SDA, whereas LA levels are unchanged, even though the LA metabolite GLA is at a higher level than the ALA metabolite SDA (Table 5). This could indicate that LA is under stronger homoeostatic regulation compared with ALA and may have implications for any future attempts to utilize C. sativa as a transgenic host for heterologous lipid metabolism. Given that any transgene-derived activities will have to efficiently interact with endogenous lipid synthesis, further biochemical characterization of C. sativa fatty acid metabolism is required. However, our initial results on the accumulation of $\Delta 6$-desaturated fatty acids in this crop support the suggestion of Lu et al., 2011 that C. sativa is an amenable host for the accumulation of non-native fatty acids, including nutritionally important ones such as SDA.

\section{Conclusions}

The results obtained in this study confirm the value of using an acyl-CoA-dependent $\Delta 6$-desaturase in attempts to synthesize C20 LC-PUFAs in transgenic plants. We have demonstrated that the use of either algal or vertebrate acyl-CoA $\Delta 6$-desaturases can enhance the flux of substrate acyl chains through the LC-PUFA biosynthetic pathway, leading to increased levels of target C20 PUFAs and reduced accumulation of undesirable intermediates such GLA. Conversely, the presence of an acyl-CoA-dependent $\Delta 6$-desaturase leads to the more uniform distribution of C20 

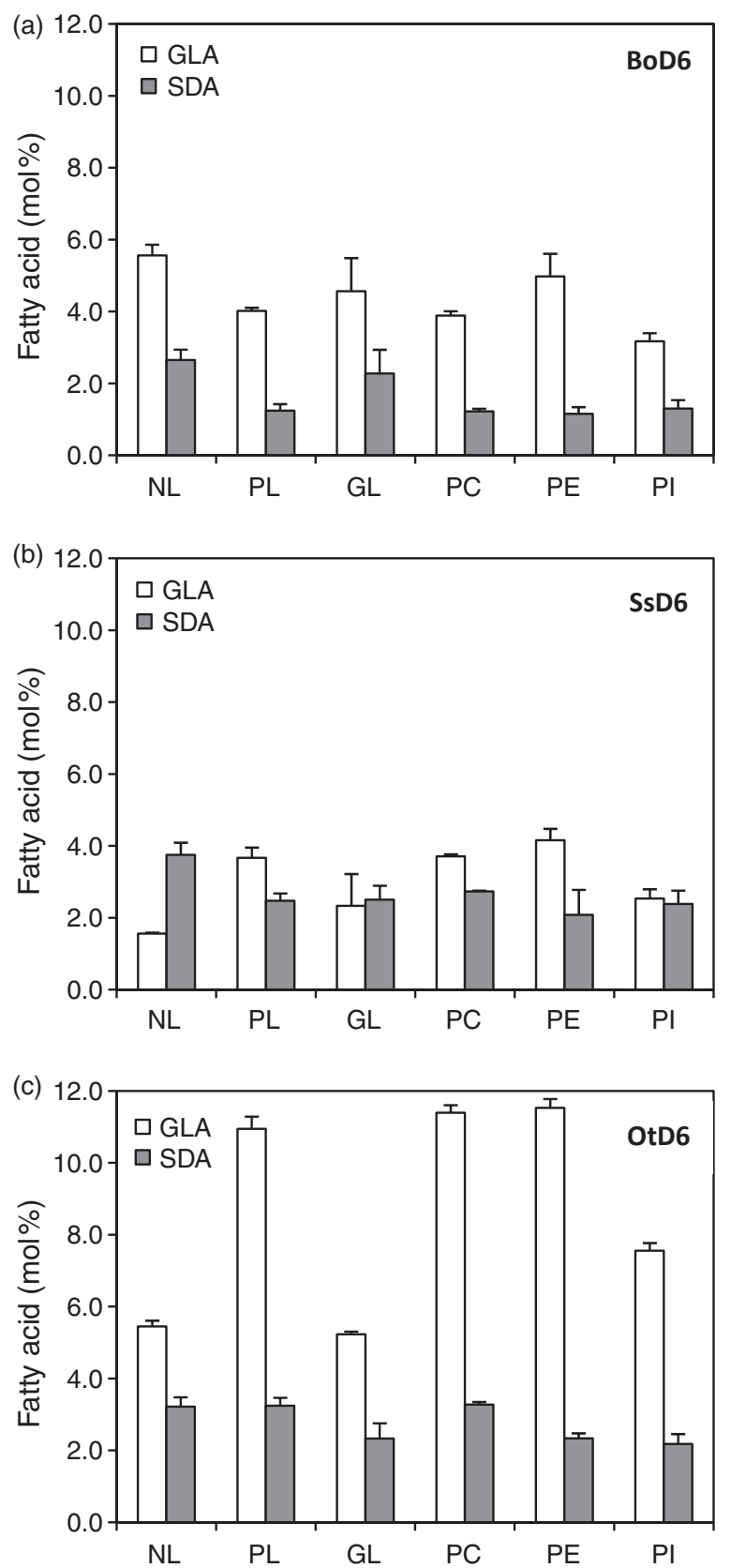

Figure 5 Distribution of GLA and stearidonic acid (SDA) within the different lipid fractions of transgenic Arabidopsis seeds, expressing different $\Delta 6$-desaturases ( $a$, BoD6; b, SsD6; c, OtD6). Mature Arabidopsis seeds were used for lipid analysis. The ratios were calculated as (products/educt) using values corresponding to per cent of total fatty acids. The values are the mean \pm SD $(<1)$ of three independent lines.

PUFAs in phospholipids and neutral lipids, commensurate with the elevated levels of C20 acyl-CoAs available as substrates for forward acylation reactions associated with the Kennedy pathway.

\section{Experimental procedures}

\section{Expression in yeast}

The different yeast expression constructs used in this study are listed in Table S2. The S. cerevisiae strains W303-1A and INVSC1 from Invitrogen were transformed with plasmid DNA using a lithium acetate method. Cultures were grown at $22^{\circ} \mathrm{C}$ in the presence of $2 \%(\mathrm{v} / \mathrm{v})$ raffinose for $48 \mathrm{~h}$, and expression of the transgenes was induced by addition of galactose to $2 \%$ in the presence of $0.5 \mathrm{~mm}$ of ALA and $1 \%(\mathrm{~W} / \mathrm{v})$ tergitol-Nonidet p40 (Sigma, St Louis, MO, USA) as described (Tonon et al., 2005).

For kinetic experiments, transgenic yeast cultures were grown for $24 \mathrm{~h}$ at $30{ }^{\circ} \mathrm{C}$ in the presence of galactose to a $1.5 \mathrm{OD}_{600}$ for permanent expression of $\Delta 6$-desaturases before the start of kinetic analysis by adding $250 \mu \mathrm{m}$ ALA as a substrate Domergue et al., 2003). Just before supplementing ALA (BFA) as well as 5 min, 1 and $24 \mathrm{~h}$ after substrate addition, the fatty acid composition of the transgenic yeast cells and of their acyl-CoAs were determined.

\section{Acyl-CoA profiling}

For acyl-CoA analysis of yeast, $20 \mathrm{~mL}$ of liquid cultures was harvested and frozen in liquid nitrogen for subsequent extraction and quantitative analysis of fluorescent acyl-etheno-CoA derivatives by HPLC (Agilent 1100 LC system; Phenomenex LUNA $150 \times 2 \mathrm{~mm} \mathrm{C18(2)} \mathrm{column)} \mathrm{as} \mathrm{described} \mathrm{previously} \mathrm{(Larson}$ and Graham, 2001).

For acyl-CoA analysis of plants, $20 \mathrm{mg}$ of developing (15 days after flowering) seeds was collected, frozen in liquid nitrogen and analyzed as described earlier.

\section{Fatty acid analysis}

Fatty acids were extracted and methylated as described (Sayanova et al., 1997). Methyl ester derivatives of total fatty acids extracted were analyzed by GC and GC-MS.

\section{Lipid analysis}

Three-hundred milligrams of seeds was heated for $10 \mathrm{~min}$ at $95^{\circ} \mathrm{C}$ in $1 \mathrm{~mL}$ of isopropanol and homogenized using a mortar and pestle. The homogenate was centrifuged; supernatant collected, and the pellet re-extracted with isopropanol/chloroform $(1: 1, v / v)$. Both extracts were pooled, evaporated and dissolved in chloroform/acetic acid (100:1, v/v). The lipid extract was loaded on a Sep-pack column and pre-fractionated into neutral lipids, glycolipids and phospholipids adding chloroform/acetic acid (100: 1, v/v), acetone/acetic acid (100:1) and methanol, respectively. These fractions were further resolved on thin-layer chromatography silica gel plates, thickness $0.25 \mathrm{~mm}$. Neutral lipids were developed with hexane/ethyl ether/formic acid (75:25:1, by volume), and polar lipids with chloroform/methanol/ammonia/water (70:30:4:1, by volume). The individual lipid classes were identified under UV light after a Primuline spray $[0.05 \%$ $(\mathrm{w} / \mathrm{v})$ in acetone/water, $80: 20, \mathrm{v} / \mathrm{v}]$, scraped from the plate and used directly for methylation or extracted for further analysis.

\section{Plant material and growth conditions}

Arabidopsis thaliana, Columbia (Col-0) ecotype, were grown for analyses in a controlled environment chamber at $23^{\circ} \mathrm{C}$ day $/ 18{ }^{\circ} \mathrm{C}$ night, $50 \%-60 \%$ humidity, and kept on a $16-\mathrm{h}$, $250 \mu \mathrm{mol} / \mathrm{m}^{2} / \mathrm{s}$, photoperiod (long day). Camelina sativa was grown under similar conditions.

\section{Plant transformation constructs}

Open reading frames encoding Otd6, SsD6, EmD5 and PSE1 were used as templates to chemically synthesize (Genscript Corporation, NJ) codon-optimized nucleotide sequences for expression in Arabidopsis. All coding regions used were placed under 
Table 5 Accumulation of $\Delta 6$-desaturated fatty acids in transgenic Camelina sativa via seed-specific expression of OtD6

\begin{tabular}{|c|c|c|c|c|c|c|c|c|c|c|}
\hline & \multirow[b]{2}{*}{ Line ID } & \multicolumn{9}{|c|}{ Fatty acids (Mol\%) } \\
\hline & & $16: 0$ & $18: 0$ & $18: 1$ & $18: 2$ & GLA & ALA & SDA & $20: 1$ & Others \\
\hline Mean & WT & 7.0 & 3.3 & 16.2 & 20.8 & 0.0 & 29.5 & 0.0 & 13.6 & 9.4 \\
\hline SD & & 0.1 & 0.5 & 0.7 & 0.4 & 0.0 & 0.4 & 0.0 & 1.2 & 0.4 \\
\hline Mean & $\# 48$ & 10.3 & 6.8 & 5.5 & 19.1 & 18.8 & 8.3 & 12.5 & 8.0 & 10.8 \\
\hline SD & & 0.3 & 0.6 & 0.2 & 0.3 & 1.0 & 0.8 & 0.5 & 0.4 & 0.9 \\
\hline Mean & \#75 & 9.7 & 9.2 & 6.2 & 20.9 & 17.5 & 7.5 & 10.5 & 7.0 & 11.6 \\
\hline SD & & 0.1 & 0.3 & 0.0 & 0.6 & 0.7 & 0.1 & 0.6 & 0.1 & 0.3 \\
\hline Mean & \#2 & 10.1 & 8.1 & 6.0 & 20.0 & 18.7 & 8.2 & 11.6 & 6.5 & 10.8 \\
\hline SD & & 0.3 & 0.5 & 0.1 & 0.1 & 0.1 & 0.5 & 0.3 & 0.3 & 0.7 \\
\hline Mean & \#206 & 8.8 & 7.1 & 6.7 & 22.0 & 16.4 & 9.8 & 10.4 & 8.0 & 10.9 \\
\hline SD & & 0.4 & 0.4 & 0.3 & 0.5 & 0.8 & 0.8 & 0.2 & 0.2 & 0.4 \\
\hline Mean & $\# 244$ & 8.8 & 7.4 & 5.6 & 19.5 & 16.6 & 10.3 & 12.3 & 7.9 & 11.5 \\
\hline SD & & 0.5 & 0.1 & 0.4 & 0.8 & 0.7 & 0.6 & 0.6 & 0.3 & 0.2 \\
\hline Mean & $\# 40$ & 10.8 & 6.7 & 5.4 & 19.0 & 19.0 & 9.0 & 13.4 & 6.9 & 9.8 \\
\hline SD & & 0.4 & 0.4 & 0.4 & 0.4 & 0.6 & 0.3 & 0.1 & 0.3 & 1.0 \\
\hline Mean & $\# 53$ & 9.9 & 7.9 & 5.7 & 19.4 & 18.7 & 8.3 & 12.2 & 7.1 & 10.8 \\
\hline SD & & 0.4 & 0.2 & 0.2 & 0.8 & 0.3 & 0.8 & 0.6 & 0.1 & 0.2 \\
\hline Mean & $\# 232$ & 9.7 & 9.0 & 6.2 & 20.5 & 17.4 & 7.8 & 10.5 & 7.4 & 11.5 \\
\hline SD & & 0.2 & 0.3 & 0.3 & 0.6 & 1.1 & 0.3 & 0.7 & 0.4 & 1.1 \\
\hline Mean & $\# 200$ & 8.9 & 8.1 & 6.4 & 19.9 & 17.9 & 8.6 & 11.5 & 7.6 & 11.1 \\
\hline SD & & 0.2 & 0.1 & 0.1 & 0.2 & 0.4 & 0.3 & 0.0 & 0.1 & 0.6 \\
\hline Mean & $\# 102$ & 9.4 & 8.0 & 5.7 & 19.3 & 18.0 & 8.7 & 12.1 & 7.7 & 11.1 \\
\hline SD & & 0.2 & 0.4 & 0.0 & 0.3 & 0.4 & 0.6 & 0.5 & 0.1 & 0.3 \\
\hline Mean & \#55 & 9.7 & 8.9 & 5.8 & 20.6 & 18.0 & 7.9 & 11.3 & 7.2 & 10.6 \\
\hline SD & & 0.3 & 0.4 & 0.0 & 0.3 & 0.2 & 0.3 & 0.3 & 0.3 & 0.4 \\
\hline Mean & $\# 215$ & 9.3 & 6.9 & 5.5 & 19.5 & 17.0 & 10.5 & 13.0 & 8.0 & 10.4 \\
\hline SD & & 0.1 & 0.8 & 0.3 & 0.9 & 0.5 & 0.8 & 0.9 & 0.1 & 0.2 \\
\hline
\end{tabular}

Total seed fatty acids from transgenic C. sativa lines (T3) expressing the OtD6 desaturase under the glycinin promoter are shown, compared with wild type controls. For each line, the figure given is the mean of three biological replicates ( 5 seeds/rep) for each line. SD is shown. The predominant fatty acids are shown

GLA, $\gamma$-linolenic acid; ALA, $\boldsymbol{\alpha}$-linolenic acid; SDA, stearidonic acid.

the control of the glycinin-1 promoter. The coding sequences for $\Delta 6$ desaturases from B. officinalis (Sayanova et al., 1997) and both Salmo salar (Zheng et al., 2005) and O. tauri (Domergue et al., 2005a, 2005b) were inserted as Xbal-Xhol and EcoRI-Xhol fragments, respectively, into the binary vector RS-3GSeedDSred, kindly provided by Dr Ed Cahoon (University of Nebraska). The coding regions of $\Delta 5$ desaturase from $E$. huxleyi (EmD5, Sayanova et al., 2011) and $\Delta 6$-elongase PSE1 from P. patens (Zank et al., 2002) were introduced as Notl-Notl fragments into pKMS3 promoter-nos terminator expression cassettes. The double-gene constructs were built by removing glycinin-1::PSE1 cassette from pKMS3 by digestion with Ascl and cloning it into Mlul site of the binary vector RS-3GSeedDSred containing one of the three $\Delta 6$-desaturases (BoD6, SsD6 or OtD6). The glycinin-1::EmD5 expression cassette was excised with AsCl and inserted into the binary vector pKAN as previously decribed (Sayanova et al., 2006). The orientation of expression cassettes was unidirectional within the T-DNA vector.

\section{Generation of transgenic plants}

Transgenic Arabidopsis plants were generated as previously described (Sayanova et al., 2006). Single-transgenic plants expressing $\Delta 6$-desaturases (BoD6, SsD6 or OtD6) and double- transgenic plants all showing positive for visual selection of the DsRed marker were self-fertilized to T3. Single-transgenic plants expressing EmD5 were kanamycin resistant and were germinated after two rounds of self-fertilization. Triple-transgenic plants were produced by transforming the double-transgenic homozygous lines with the highest C20 fatty acid content with a T-DNA containing EmD5 and a kanamycin-resistance gene. Red seeds from kanamycin $(50 \mu \mathrm{g} / \mathrm{mL})$ resistant plants were selected and grown to obtain homozygous T3 transformants.

Transgenic Camelina sativa plants were generated, selected and grown as described by Lu and Kang (2008). Samples analyzed in this study were T3 generation.

\section{Acknowledgements}

Rothamsted Research receives grant-aided support from the BBSRC (UK). The authors acknowledge the contributions of BASF Plant Sciences.

\section{References}

Abbadi, A., Domergue, F., Bauer, J., Napier, J.A., Welti, R., Zähringer, U., Cirpus, P. and Heinz, E. (2004) Biosynthesis of very-long-chain 
polyunsaturated fatty acids in transgenic oilseeds: constraints on their accumulation. Plant Cell, 16, 2734-2748.

Beaudoin, F., Michaelson, L.V., Hey, S.J., Lewis, M.J., Shewry, P.R., Sayanova, O. and Napier, J.A. (2000) Heterologous reconstitution in yeast of the polyunsaturated fatty acid biosynthetic pathway. Proc. Natl Acad. Sci. USA, 97, 6421-6426.

Cheng, B., Wu, G., Vrinten, P., Falk, K., Bauer, J. and Qiu, X. (2010) Towards the production of high levels of eicosapentaenoic acid in transgenic plants: the effects of different host species, genes and promoters. Transgenic Res. 19, 221-229.

Cho, H.P., Nakamura, M.T. and Clarke, S.D. (1999) Cloning, expression, and nutritional regulation of the mammalian $\Delta 6$ desaturase. J. Biol. Chem. 274, 471-477.

Damude, H.G. and Kinney, A.J. (2008) Enhancing plant seed oils for human nutrition. Plant Physiol. 147, 962-968.

Domergue, F., Abbadi, A., Ott, C., Zank, T.K., Zähringer, U. and Heinz, E. (2003) Acyl carriers used as substrates by the desaturases and elongases involved in very long-chain polyunsaturated fatty acids biosynthesis reconstituted in yeast. J. Biol. Chem. 278, 35115-35126.

Domergue, F., Abbadi, A. and Heinz, E. (2005a) Relief for fish stocks: oceanic fatty acids in transgenic oilseeds. Trends Plant Sci. 10, 112-116.

Domergue, F., Abbadi, A., Zähringer, U., Moreau, H. and Heinz, E. (2005b) In vivo characterization of the first acyl-CoA $\Delta 6$-desaturase from a member of the plant kingdom, the microalga Ostreococcus tauri. Biochem. J. 389, 483-490.

Drexler, H., Spiekermann, P., Meyer, A., Domergue, F., Zank, T., Sperling, P., Abbadi, A. and Heinz, E. (2003) Metabolic engineering of fatty acids for breeding of new oilseed crops: strategies, problems and first results. J. Plant Physiol. 160, 779-802.

Eckert, H., LaVallee, B., Schweiger, B.J., Kinney, A.J., Cahoon, E.B. and Clemente, T. (2006) Co-expression of the borage $\Delta 6$ desaturase and the Arabidopsis $\Delta 15$ desaturase results in high accumulation of stearidonic acid in the seeds of transgenic soybean. Planta, 224, 1050-1057.

Gehringer, A., Friedt, W., Lühs, W. and Snowdon, R.J. (2006) Genetic mapping of agronomic traits in false flax (Camelina sativa subsp. sativa). Genome, 49, 1555-1563.

Griffiths, G., Stobart, A.K. and Stymne, S. (1988) $\Delta 6$ - and $\Delta 12$-desaturase activities and phosphatidic acid formation in microsomal preparations from the developing cotyledons of common borage (Borago officinalis). Biochem. J. 252, 641-647.

Hoffmann, M., Wagner, M., Abbadi, A., Fulda, M. and Feussner, I. (2008) Metabolic engineering of omega3-very long chain polyunsaturated fatty acid production by an exclusively acyl-CoA-dependent pathway. J. Biol. Chem. 283, 22352-22362.

Hong, H., Datla, N., Reed, D.W., Covello, P.S., MacKenzie, S.L. and Qiu, X. (2002) High-level production of $\gamma$-linolenic acid in Brassica juncea using a $\Delta 6$ desaturase from Pythium irregulare. Plant Physiol. 129, 354-362.

Jackson, F.M., Fraser, T.C., Smith, M.A., Lazarus, C., Stobart, A.K. and Griffiths, G. (1998) Biosynthesis of C18 polyunsaturated fatty acids in microsomal membrane preparations from the filamentous fungus Mucor circinelloides. Eur. J. Biochem. 252, 513-519.

Larson, T.R. and Graham, I.A. (2001) A novel technique for the sensitive quantification of acyl CoA esters from plant tissues. Plant J. 25, 115-125.

Lu, C. and Kang, J. (2008) Generation of transgenic plants of a potential oilseed crop Camelina sativa by Agrobacterium-mediated transformation. Plant Cell Rep. 27, 273-278.

Lu, C., Napier, J.A., Clemente, T.E. and Cahoon, E.B. (2011) New frontiers in oilseed biotechnology: meeting the global demand for vegetable oils for food, feed, biofuel, and industrial applications. Curr. Opin. Biotechnol. 22, 252-259.

Marquardt, A., Stöhr, H., White, K. and Weber, B.H. (2000) cDNA cloning, genomic structure, and chromosomal localization of three members of the human fatty acid desaturase family. Genomics, 66, 175-183.

Napier, J.A. (2007) The production of unusual fatty acids in transgenic plants. Annu. Rev. Plant Biol. 58, 295-319.

Napier, J.A. and Graham, I.A. (2010) Tailoring plant lipid composition: designer oilseeds come of age. Curr. Opin. Plant Biol. 13, 330-337.
Napier, J.A. and Michaelson, L.V. (2001) Genomic and functional characterization of polyunsaturated fatty acid biosynthesis in Caenorhabditis elegans. Lipids, 36, 761-766.

Napier, J.A., Michaelson, L.V. and Sayanova, O. (2003) The role of cytochrome b5 fusion desaturases in the synthesis of polyunsaturated fatty acids. Prostaglandins Leukot. Essent. Fatty Acids 68, 135-143.

Napier, J.A., Sayanova, O., Qi, B. and Lazarus, C.M. (2004) Progress toward the production of long-chain polyunsaturated fatty acids in transgenic plants. Lipids, 39, 1067-1075.

Okayasu, T., Nagao, M., Ishibashi, T. and Imai, Y. (1981) Purification and partial characterization of linoleoyl-CoA desaturase from rat liver microsomes. Arch. Biochem. Biophys. 206, 21-28.

Petrie, J.R., Shrestha, P., Mansour, M.P., Nichols, P.D., Liu, Q. and Singh, S.P. (2010) Metabolic engineering of omega-3 long-chain polyunsaturated fatty acids in plants using an acyl-CoA $\Delta 6$-desaturase with omega3-preference from the marine microalga Micromonas pusilla. Metab. Eng. 12, 233-240.

Robert, S.S. (2006) Production of eicosapentaenoic and docosahexaenoic acid-containing oils in transgenic land plants for human and aquaculture nutrition. Mar. Biotechnol. (NY) 8, 103-109.

Robert, S.S., Singh, S.P., Zhou, X.-R., Petrie, J.R., Blackburn, S.I., Mansour, P.M., Nichols, P.D., Liu, Q. and Green, A.G. (2005) Metabolic engineering of Arabidopsis to produce nutritionally important DHA in seed oil. Funct. Plant Biol. 32, 473-479.

Sato, S., Xing, A., Ye, X., Schweiger, B., Kinney, A.J., Graef, G. and Clemente, T.E. (2004) Production of $\gamma$-linolenic acid and stearidonic acid in seeds of marker-free transgenic soybean. Crop Sci. 44, 646-652.

Sayanova, O., Smith, M.A., Lapinskas, P., Stobart, A.K., Dobson, G., Christie, W.W., Shewry, P.R. and Napier, J.A. (1997) Expression of a borage desaturase cDNA containing an N-terminal cytochrome $b_{5}$ domain results in the accumulation of high levels of $\Delta 6$-desaturatedfatty acids in transgenic tobacco. Proc. Natl Acad. Sci. USA, 94, 4211-4216.

Sayanova, O., Haslam, R., Venegas-Calerón, M. and Napier, J.A. (2006) Identification of Primula "front-end" desaturases with distinct $n-6$ or n-3 substrate preferences. Planta, 224, 1269-1277.

Sayanova, O., Haslam, R.P., Calerón, M.V., López, N.R., Worthy, C., Rooks, P., Allen, M.J. and Napier, J.A. (2011) Identification and functional characterisation of genes encoding the omega-3 polyunsaturated fatty acid biosynthetic pathway from the coccolithophore Emiliania huxleyi. Phytochemistry, 72, 594-600.

Sperling, P., Linscheid, M., Stöcker, S., Mühlbach, H.P. and Heinz, E. (1993) In vivo desaturation of cis- $\Delta 9$-monounsaturated to cis- $\Delta 9,12$-diunsaturated alkenylether glycerolipids. J. Biol. Chem. 268, 26935-26940.

Sprecher, H. and Chen, Q. (1999) Polyunsaturated fatty acid biosynthesis: a microsomal-peroxisomal process. Prostaglandins Leukot. Essent. Fatty Acids 60, 317-321.

Stymne, S. and Stobart, A.K. (1986) Biosynthesis of $\gamma$-linolenic acid in cotyledons and microsomal preparations of the developing seeds of common borage (Borago officinalis). Biochem. J. 240, 385-393.

Tonon, T., Sayanova, O., Michaelson, L.V., Qing, R., Harvey, D., Larson, T.R., Li, Y., Napier, J.A. and Graham, I.A. (2005) Fatty acid desaturases from the microalga Thalassiosira pseudonana. FEBS J. 272, 3401-3412.

Tripodi, K.E., Buttigliero, L.V., Altabe, S.G. and Uttaro, A.D. (2006) Functional characterization of front-end desaturases from trypanosomatids depicts the first polyunsaturated fatty acid biosynthetic pathway from a parasitic protozoan. FEBS J. 273, 271-280.

Venegas-Calerón, M., Sayanova, O. and Napier, J.A. (2010) An alternative to fish oils: metabolic engineering of oil-seed crops to produce omega-3 long chain polyunsaturated fatty acids. Prog. Lipid Res. 49, 108-119.

Zank, T.K., Zähringer, U., Beckmann, C., Pohnert, G., Boland, W., Holtorf, H., Reski, R., Lerchl, J. and Heinz, E. (2002) Cloning and functional characterisation of an enzyme involved in the elongation of $\Delta 6$-polyunsaturated fatty acids from the moss Physcomitrella patens. Plant J. 31, 255-268.

Zheng, X., Tocher, D.R., Dickson, C.A., Bell, J.G. and Teale, A.J. (2005) Highly unsaturated fatty acid synthesis in vertebrates: new insights with the cloning and characterization of a delta6 desaturase of Atlantic salmon. Lipids, 40, 13-24. 


\section{Supporting information}

Additional Supporting information may be found in the online version of this article:

Figure S1 Kinetic analysis of fatty acid profiles of yeast expressing $\Delta 6$-desaturases in the presence of 18:3 n-3.

Table S1 Kinetic analysis of fatty acid profiles in lipids and acylCoAs of yeast co-expressing $\Delta 6$-desaturases and CeElo6 in the presence of ALA.
Table S2 Description of yeast strains used in this study.

Please note: Wiley-Blackwell are not responsible for the content or functionality of any supporting materials supplied by the authors. Any queries (other than missing material) should be directed to the corresponding author for the article. 